\title{
Thermal explosions in spherical vessels at large Rayleigh numbers
}

\author{
I. Iglesias ${ }^{\mathrm{a}}$, D. Moreno-Boza ${ }^{\mathrm{b}}$, A.L. Sánchez ${ }^{\mathrm{b}, *}$, A. Liñán $^{\mathrm{c}}$, F.A. Williams ${ }^{\mathrm{b}}$ \\ a Departamento de Ingeniería Térmica y de Fluidos, Universidad Carlos III de Madrid, Leganés 28911, Spain \\ ${ }^{\mathrm{b}}$ Department of Mechanical and Aerospace Engineering, University of California San Diego, La Jolla, CA 92093-0411, USA \\ ${ }^{\mathrm{c}}$ ETSI Aeronáuticos, Pl. Cardenal Cisneros 3, Madrid 28040, Spain
}

\section{A R T I C L E I N F O}

\section{Article history:}

Received 24 June 2017

Received in revised form 22 August 2017

Accepted 28 August 2017

Available online 5 September 2017

\section{MSC:}

00-01

$99-00$

Keywords:

Thermal explosions

Laminar reacting flows

Natural convection

Boundary-layer theory

\begin{abstract}
A B S T R A C T
This paper investigates effects of buoyancy-driven motion on the "slowly reacting" mode of combustion, and its thermal-explosion limits, of an initially cold gaseous mixture enclosed in a spherical vessel with a constant wall temperature. As in Frank-Kamenetskii's seminal analysis, the strong temperature dependence of the effective overall reaction is modeled with a single irreversible reaction with an Arrhenius rate having a large activation energy. Besides the classical Damköhler number $D a$, measuring the ratio of the heat-release rate by chemical reaction evaluated at the wall temperature to the rate of heat removal by heat conduction to the wall, the solution is seen to depend on the Rayleigh number $R a$, measuring the effect of buoyancy-induced motion on the heat-transport rate. For values of $D a$ below a critical value $D a_{c}$ the system evolves in a slowly reacting mode where the heat losses to the wall limit the temperature increase associated with the chemical reaction, whereas for $D a>D a_{c}$ the initial stage of slow reaction ends abruptly at a well-defined ignition time, at which a thermal runaway occurs. Transient numerical integrations of the initial stage of slow reaction, formulated in the distinguished limit $D a \sim 1$ and $R a \sim 1$ with account taken of the effects of the temporal pressure variation, are used to investigate influences of natural convection on thermal-explosion development, including changes in ignition times for $D a>D a_{c}$ and modified explosion curves. Our analysis reveals that Frank-Kamenetskii's criterion for the determination of critical explosion conditions, based on the investigation of existence of steady solutions, provides values of $D a_{c}(R a)$ that are identical to those extracted from the transient computations. Specific consideration is given to the structure of the steady solution in the asymptotic limit $R a \gg 1$ in which the flow includes a thin chemically frozen near-wall boundary layer of downward moving cold gas bounding a central inviscid region of slowly rising reacting flow driven by the boundary-layer entrainment, with the critical explosion conditions predicted to occur for $D a_{c} \sim R a^{1 / 4}$. The mathematical structure of the resulting boundary-layer problem is fundamentally similar to that found in the unrelated problem of flow in curved pipes at large Dean numbers. As in that similar problem, the boundary layer exhibits a region of recirculating flow, so that the problem must be formulated as a boundary-value problem accounting for the self-similar local solutions that exist near the upper and lower stagnation points. The problem is solved with use made of an approximate integral method. The resulting asymptotic prediction for the critical Damköhler number $D a_{c}=0.655 R a^{1 / 4}$ is found to be in excellent agreement with the results obtained by integration of the complete conservation equations for $R a \gg 1$.
\end{abstract}

(c) 2017 Elsevier Ltd. All rights reserved.

\section{Introduction}

In developing the excellent stationary theory of thermal explosions [1], Frank-Kamenetskii (FK) investigated the role of the strong temperature sensitivity of the reaction rate in the combustion of enclosed reactive mixtures. Explosion limits were defined as the critical conditions for existence of a steady, slowly reacting

\footnotetext{
* Corresponding author.

E-mail address: als@ucsd.edu (A.L. Sánchez).
}

mode of combustion in which the heat-conduction losses to the wall limit the temperature rise in such a way that the reaction rate does not exceed in order of magnitude its near-wall value. With the constant-density approximation used in the early work, heat transfer to the wall occurred only by conduction, its effect being measured by a Damköhler number $D a$, defined below in (3) as the ratio of the time of heat conduction to the wall to the homogeneous thermal-explosion time. The curve giving the variation of the peak temperature with $\mathrm{Da}$ exhibits a first turning point at a critical value $D a=D a_{c}$ of order unity (e.g., $D a_{c}=3.32$ for spherical 
containers), which identifies the explosion limit in FK theory. Using the value of $D a_{c}$ together with the definition of the Damkhöhler number leads to explicit expressions for critical explosion sizes, giving results in close agreement with experimental observations, a remarkable achievement of the early theory, given the many different simplifying assumptions involved in its derivation [2]. This success has motivated recent extensions of the early theory incorporating realistic chemistry in descriptions of hydrogen-oxygen systems that have been shown to predict explosion conditions in spherical vessels in excellent agreement with experiments [3], including critical pressures along the so-called third-explosion limit [4]. The FK problem was recently revisited in [5] to investigate influences on ignition times of initial conditions and of temporal pressure variations in spherical vessels with fixed walls.

For gaseous mixtures under normal gravity the small temperature differences characterizing the slowly reacting mode of combustion are known to generate a convective motion that promotes heat transfer to the wall, measured by a relevant Rayleigh number $R a$, defined below in (4). Influences of buoyancy on the resulting critical Damköhler numbers, discussed in early references [2], have been investigated numerically for simplified vessel geometries, as referenced below, including the infinite slab, the infinite horizontal cylinder, and the sphere, the last one being the configuration considered in the present paper.

The problem for a slab configuration, with a reactive gas bounded by two isothermal infinite horizontal walls, has been addressed in numerous theoretical analyses [6-10]. As in the closely related case of the Rayleigh-Bénard problem of convection produced by wall-temperature differences, a motionless quasi-steady combustion mode may exist for values of the Rayleigh number below a critical value, at which a bifurcation occurs to a convective state, as described by linear stability analyses $[6,7]$. A noteworthy feature of the slab geometry is that, contrary to widespread expectations, convection does not always hinder the development of the thermal explosion [8]. While sufficiently small eddies with widths on the order of or smaller than the slab height raise the explosion threshold by enhancing heat removal from the reaction region, long-wave convection rolls favor explosions by promoting the formation of hot spots [8], an aspect of the problem further investigated in [9] with use made of a prescribed velocity field. The closely related problem of a horizontal cylindrical container of square section with adiabatic vertical walls has been also considered by different researchers [11-13]. For this geometry, the critical Damkhöhler number $D a_{c}$ was found to increase in the presence of buoyancy-induced motion [11]. The rich nonlinear dynamics of weakly reactive solutions for subcritical values of the Damkhöhler number $D a<D a_{c}$ has now been investigated in more recent bifurcation analyses [12,13].

Unlike the horizontal infinite slab, for reactive gases in cylindrical and spherical vessels any gravity force at all establishes buoyant motion because the horizontal variations of the density that are present in these configurations generate forces that cannot be counteracted by a vertical pressure gradient. The case of a horizontal circular cylinder has been investigated both experimentally [11] and numerically [14]. This last work includes transient computations for Rayleigh numbers up to $10^{4}$, for different initial temperatures both above and below the wall value. Influences of buoyancy on thermal explosions in spherical vessels have also been considered in the past. In connection with the present work, the most closely related investigations are the numerical computations of Hayhurst and coworkers [15-17]. To enable comparisons with previous experimental results [18] to be made, the thermal decomposition of azomethane vapor was considered in [15] when selecting the specific chemical-rate parameters, for values of the Rayleigh numbers up to 21900 . Resulting values of $D a_{c}$ were found to differ by only a few percent from those of the buoyancy-free predictions for values of $R a$ as large as a few hundred, in agreement with earlier results $[10,11,14]$. Larger values of the Rayleigh numbers were considered in subsequent direct numerical simulations $[16,17]$ addressing transient problems with the initial gas temperature equal to the vessel wall temperature. The flow was found to remain laminar for values of $R a<10^{6}$. More recent numerical work by this same research group includes investigation of combined natural and forced convection on thermal explosions [19] as well as consideration of non-isothermal walls by use of a Robin condition including a heat-transfer coefficient [20].

The remarkably small influence of buoyancy on the critical ignition Damkhöhler number $D a_{c}$ for moderate values of $R a$ was studied recently by asymptotic methods [21]. At leading order in the limit $R a \ll 1$, the analysis revealed that the spherically symmetrical temperature field corresponding to the buoyancy-free FK solution is accompanied by a toroidal vortex, which may be called the Frank-Kamenetskii vortex, resulting from the balance between buoyancy and viscous forces. The modifications to the explosion curve, determined by extending the perturbation analysis to a higher order, were found to scale with $R a^{2}$, but the numerical factors in the expansion for $D a_{c}$ are extremely small, so that the corrections associated with buoyancy remain smaller than $10 \%$ even at $R a=300$.

While this previous analysis showed that effects of weak buoyancy associated with values of Ra on the order of a few hundred can be suitably addressed by asymptotic methods, the description of more pronounced buoyancy effects for larger values of $R a$ requires numerical integrations. Results of relevant integrations are reported below for values of the Rayleigh number up to $R a=7 \times 10^{6}$, thereby covering the entire range of flow conditions under which the flow may be expected to remain laminar. Besides numerical integrations, perturbation methods are used to investigate the flow structure that develops in the asymptotic limit $R a \gg 1$, which is shown here to include a thin chemically frozen boundary layer driven by buoyancy, surrounding the vessel surface, and an inner chemically reacting inviscid region. The analysis at leading order provides the formulation of a boundary-value problem that determines the rescaled FK explosion curve, including the critical explosion conditions. The solution is obtained by an approximate method involving an integral form of the boundary-layer equations, which is found to produce predictions for the critical Damköhler number that compare favorably with the results of numerical integrations.

The presentation begins in Section 2 with the complete set of equations for the slowly reacting mode of combustion with natural convection, with account taken of the nonnegligible effect of the temporal pressure variations in the energy equation. Results of steady and transient computations are presented in Section 3, including the associated FK predictions for the thermal explosion limits in the presence of buoyancy-driven flow. The asymptotic limit $R a \gg 1$ is considered next, in Section 4. The paper ends in Section 5 with concluding remarks.

\section{Formulation for the transient slowly reacting mode of combustion in the presence of buoyancy}

The analysis considers a spherical vessel of radius $a$ filled with a reactant ideal-gas mixture at a strictly uniform temperature $T_{\mathrm{I}}$, assumed to be sufficiently low for the chemical reaction to be initially negligible. In order to promote reaction, the wall temperature is rapidly increased at the initial instant of time to a fixed constant value $T_{o}>T_{\mathrm{I}}$. This temperature rise is assumed to occur in a time that is short compared with the heat-conduction time across the 
vessel but still much larger than the characteristic acoustic time, so that spatial pressure variations remain very small. The initial reactant mass fraction $Y_{\mathrm{I}}$ is uniform, and any changes in the average molecular weight of the gas are considered to be negligible. The initial average gas density $\rho_{\mathrm{I}}$ is the ratio of the mass of gas present in the chamber to the chamber volume, and the initial average pressure $p_{\mathrm{I}}$ is determined from $T_{\mathrm{I}}$ and $\rho_{\mathrm{I}}$ by application of the ideal gas law. Initial pressure variations of the gas, at rest in hydrostatic equilibrium, are extremely small for chambers of sizes of interest in the Earth's gravitational field and give rise to correspondingly small density variations in the isothermal gas. The average pressure $p_{o}$ is defined by employing $\rho_{\mathrm{I}}$ and $T_{o}$ in the ideal gas law.

As in Frank-Kamenetskii's work [1], an overall Arrhenius reaction is adopted here, with the mass of reactant consumed per unit volume per unit time $\dot{m}$ given by

$\dot{m} / \rho=k(T) Y=Y B \exp [-E /(R T)]$,

where $\rho$ is the gas density, $T$ its temperature, and $Y$ is the reactant mass fraction. The temperature-dependent reaction-rate constant $k=B \exp [-E /(R T)]$ includes the frequency factor $B$, the activation energy $E$, and the universal gas constant $R$. In this simple chemistry description, the heat-release rate per unit volume is $q \dot{m}$, where $q$ denotes the amount of heat released per unit mass of reactant consumed. The ratio of the heat released per unit mass of gas mixture $q Y_{\mathrm{I}}$ to the gas enthalpy evaluated at the wall temperature $c_{p} T_{o}$, with $c_{p}$ denoting the specific heat at constant pressure, defines the exothermicity parameter $\alpha=\left(q Y_{I}\right) /\left(c_{p} T_{o}\right)$, of order unity or larger in applications.

The formulation of the transient thermal-explosion problem follows our earlier work [21]. The nondimensional activation energy $\beta=E /\left(R T_{o}\right)$ based on the wall temperature $T_{o}$ is assumed to be large. As a result, small temperature increments $T-T_{0}$, of the order of the FK temperature $T_{o} / \beta$, suffice to increase the reaction rate (1) by a factor of order unity. The associated chemical time needed to increase the enthalpy by an amount of order $c_{p} T_{o} / \beta$ is given by

$t_{e}=\frac{1}{\alpha \beta B \exp \left[-E /\left(R T_{o}\right)\right]}$.

This time is compared with the characteristic heat-conduction time across the vessel $t_{c}=a^{2} / D_{T}$ in defining the FK Damköhler number,

$D a=\frac{t_{c}}{t_{e}}=\frac{a^{2}}{D_{T}} \frac{q Y_{\mathrm{I}}}{c_{p} T_{o}} \frac{E}{R T_{o}} B \exp \left[-E /\left(R T_{o}\right)\right]$,

where $D_{T}$ is the thermal diffusivity. The other important parameter in the description is the Rayleigh number (based on the ordering of the temperature difference),

$R a=\frac{\beta^{-1} g a^{3}}{v D_{T}}$,

where $g$ is the acceleration of gravity and $v$ is the kinematic viscosity. This Rayleigh number will be seen to measure the convective transport in the energy equation, while the Grashof number, $G r=R a / P r=\beta^{-1} g a^{3} / v^{2}$, involving the Prandtl number $\operatorname{Pr}=v / D_{T}$, similarly measures convective transport in the momentum equation.

In the distinguished regime $D a \sim 1$ with $\beta \gg 1$ and $\alpha \sim 1$ considered here, variations of the temperature of order $T-T_{o} \sim T_{o} / \beta$ are expected to develop everywhere across the vessel after a transient period, scaling with the heat-conduction time $t_{c}$, during which the gas temperature increases from $T_{\mathrm{I}}$ to $T_{0}$. For values of $D a$ larger than a critical value $D a_{c}(R a)$ this transient stage ends with the development of a thermal explosion, characterized by a localized rapid increase of the temperature $T-T_{o} \sim \alpha T_{0}$ at a well-defined ignition time, followed by propagation of a premixed flame from the ignition kernel. By way of contrast, for subcritical values $D a<D a_{c}$ the temperature evolves to approach a quasisteady distribution, with $T-T_{o} \sim T_{o} / \beta$, resulting from a balance between the heat released by the chemical reaction in the vessel interior and the heat losses to the wall.

In the following non-dimensional formulation lengths are scaled with $a$, time $t$ with $t_{c}=a^{2} / D_{T}$, and velocity $\boldsymbol{v}$ with $v_{g}=\beta^{-1} g a^{2} / v$, the characteristic value resulting from a balance between the viscous and buoyancy forces, associated with density variations of order $\rho_{\mathrm{I}} / \beta$. Dimensionless variables $\phi=\beta\left(T-T_{o}\right) / T_{o}$ and $\hat{\rho}=\beta\left(\rho-\rho_{\mathrm{I}}\right) / \rho_{\mathrm{I}}$ are introduced for the temperature and density, as is appropriate for studying the slowly reacting mode of combustion. As noted in $[5,21]$, in rigid confined vessels the changes of the density are restricted to ensure that the mean spatial value of the density remains equal to $\rho_{\mathrm{I}}$, resulting in temporal variations of the pressure of order $p_{o} / \beta$, much larger than the spatial variations of the pressure associated with the low-Machnumber buoyancy-induced gas motion. These temporal changes of the pressure, which had been overlooked in previous numerical studies [15-17], are taken into account here when writing the energy balance and the equation of state with use made of the order-unity variable $\hat{p}(t)=\beta\left(p-p_{o}\right) / p_{o}$.

Only laminar solutions symmetric with respect to the vertical axis are considered in the following analysis. Cylindrical dimensionless coordinates are employed in the description, with $r$ and $z$ denoting the distance to the vertical axis and the distance to the equatorial plane, respectively, and $v_{r}$ and $v_{z}$ being the corresponding radial and vertical velocity components. The amount of reactant consumed during the transient stage considered here, corresponding to times of order $t_{c} \sim t_{e}$, is $\left(Y_{\mathrm{I}}-Y\right) / Y_{\mathrm{I}} \sim(\alpha \beta)^{-1} \ll 1$, so that reactant consumption can be neglected in the first approximation when computing ignition times $t_{i}$ and critical Damköhler numbers $D a_{c}$. Correspondingly, the equations describing the evolution of temperature and velocity reduce to

$\nabla \cdot \boldsymbol{v}=0$

$\frac{1}{\operatorname{Pr}}\left(\frac{\partial \boldsymbol{v}}{\partial t}+R a \boldsymbol{v} \cdot \nabla \boldsymbol{v}\right)=\nabla^{2} \boldsymbol{v}-\nabla p^{\prime}+\phi \mathbf{e}_{z}$,

$\frac{\partial \phi}{\partial t}-\frac{\gamma-1}{\gamma} \frac{\mathrm{d} \hat{p}}{\mathrm{~d} t}+R a \boldsymbol{v} \cdot \nabla \phi=\nabla^{2} \phi+D a \mathrm{e}^{\phi}$,

where the FK linearization $\mathrm{e}^{\phi /(1+\phi / \beta)} \simeq \mathrm{e}^{\phi}$ has been adopted for the Arrhenius exponent of the reaction-rate constant, as is appropriate in the limit $\beta \gg 1$. Additional symbols that appear in (6) and (7) are defined in the following paragraph.

In this near-explosion regime, associated with small density differences of order $\rho_{\mathrm{I}} / \beta$, the contribution of the temporal density variation to mass conservation gives a higher-order correction, of order $(\beta R a)^{-1}$, which has been neglected in (5). Also, leaving out terms of order $1 / \beta$, variations of transport coefficients and specific heat from their values evaluated for $T=T_{o}$ and $\rho=\rho_{\mathrm{I}}$ have been omitted in (6) and (7). In the momentum Eq. (6), $\mathbf{e}_{\mathbf{z}}$ is a unit vector pointing upwards (against gravity), and $p$ ' represents the pressure differences from the hydrostatic value that would apply when $\phi=0$, scaled with $\rho_{\mathrm{I}} g a / \beta$; these are essential for establishing the fluid motion, even though the magnitudes of the associated pressure changes are small compared with the uniform temporal variations of the pressure. The problem thus defined depends on the gas properties through the Prandtl number $\mathrm{Pr}$ appearing in (6) and the specific-heat ratio $\gamma$, which is present in the term arising from the time derivative of the pressure in the energyconservation Eq. (7). The values $\operatorname{Pr}=0.7$ and $\gamma=1.4$ are adopted for all computations performed below, an appropriate selection for most fuel-air combustion mixtures. 
The above equations are supplemented by the equation of state written in the linearized form

$\hat{\rho}+\phi=\hat{p}(t)$.

To ensure that the spatially mean density remains $\rho_{\mathrm{I}}$, as it must, the time-dependent pressure perturbation $\hat{p}$ must obey the relation

$\hat{p}=\bar{\phi}=\int_{-1}^{+1}\left(\int_{0}^{\sqrt{1-z^{2}}} \frac{3}{2} \phi r d r\right) \mathrm{d} z$

involving the non-zero mean value $\bar{\phi}$ of the non-dimensional temperature increase.

Inside the spherical vessel of constant wall temperature, the slowly reacting mode of combustion accounting for buoyancyinduced motion is expected to have analytical solutions of (5)-(8), bounded and symmetric with respect to the vertical axis $r=0$, satisfying the boundary conditions

$\boldsymbol{v}=\phi=0$

at the vessel wall $r^{2}+z^{2}=1$. Computation of the transient evolution requires specification of the initial conditions. In the following, we consider an initially cold stagnant gas mixture at temperature $T_{I}$ such that $T_{o}-T_{I} \sim T_{o} / \beta$, yielding the initial conditions

$\boldsymbol{v}=\phi-\phi_{I}=0$ at $t=0$,

with $\phi_{I}=\beta\left(T_{I}-T_{0}\right) / T_{0}<0$. As discussed in [5], the analysis becomes somewhat more complicated when the wall-temperature rise is larger, such that $T_{0}-T_{I} \sim T_{0}$, in which case the heating of the gas by heat conduction from the wall, which proceeds initially with negligible chemical reaction, involves relative variations of the density, temperature, and pressure of order unity.

\section{Selected numerical results}

As previously mentioned, the evolution of the reactive flow in the vessel depends fundamentally on the Damkhöhler number. If $D a$ is smaller than a critical value $D a_{c}$, larger for larger $R a$, the temperature and velocity evolve to approach a steady distribution for moderately large values of $t$, whereas for $D a>D a_{c}$ the transient stage ends with a thermal runaway at a finite ignition time. The determination of $D a_{c}$ for a given $R a$ based on transient computations requires consideration of increasing values of $D a$ until critical conditions are achieved, as was done previously in [15-17]. Alternatively, the explosion limit for a given $R a$ can be obtained directly by investigating the existence of steady solutions for the slowly reacting mode of combustion, with the value of $D a_{c}$ corresponding to the turning point of the stationary explosion curve giving the variation of the peak temperature with $D a$, that being the criterion proposed in Frank-Kamenetskii's original theory. We shall see below that both computational approaches lead to predictions of explosion limits that are virtually identical.

\subsection{FK analysis of thermal-explosion limits}

We begin by considering steady FK solutions, described by integrating the steady version of (5)-(7) supplemented by (9) with the boundary conditions (10) at the vessel wall. A standard iterative root-finding technique (Newton-Raphson) is employed in the integrations. The linearized system of equations is solved using the software package FreeFem++ [22], which employs finite elements for the spatial discretization. A summary of the steady solutions obtained for different values of $D a$ and $R a$ is given in Fig. 1.

The upper plot in Fig. 1 shows explosion diagrams (i.e., curves representing the peak temperature $\phi_{\max }$ as a function of the
Damköhler number $\mathrm{Da}$ ) for six different values of the Rayleigh number, including the classical FK results corresponding to $R a=0$, for which the turning point occurs at $D a_{\mathrm{FK}} \simeq 3.32$ with $\phi_{\max } \simeq 1.61$ [2]. Because of the enhanced heat transfer associated with the convective motion, the turning points of the resulting curves occur at larger values of $D a_{c}$ for increasing $R a$. This variation is represented in the intermediate plot of Fig. 1. As expected from previous analyses, the departures $D a_{c}-D a_{\mathrm{FK}}$ of the critical Damköhler number from the buoyancy-free value $D a_{\mathrm{FK}}$ remain fairly small for values of $R a$ on the order of a few hundred. For these conditions of weak convective motion the asymptotic prediction $D a_{c}=D a_{\mathrm{FK}}\left(1+\delta_{2} R a^{2}\right)$, derived previously in the limit $R a \ll 1$ [21], agrees well with the numerical results. The quantitative effect of buoyancy on the explosion boundary becomes clearly noticeable for $R a \gtrsim 500$, for which the departures $D a_{c}-D a_{\mathrm{FK}}$ become of order unity. The monotonic growth of $D a_{c}$ with $R a$ continues for increasing $R a$. As seen in the plot, for sufficiently large values of $R a \gtrsim 10^{4}$, the curve $D a_{c}(R a)$ displays the asymptotic behavior $D a_{c} \propto R a^{1 / 4}$, corresponding to a straight line with slope $1 / 4$ in the log-log scale used in the figure. The dashed curve $D a_{c}=\Lambda_{c} R a^{1 / 4}$ in the plot is the asymptotic prediction for $R a \gg 1$, to be derived later in Section 4 .

The motion induced by buoyancy and its associated effects on the temperature field are investigated on the bottom plots of Fig. 1 by representing the isotherms (left half of each circular plot) and streamlines (right half) corresponding to the solutions at the turning points of the six different explosion curves. A total of ten isocurves of temperature and stream function are shown in each subplot. The isotherms are equally spaced between the value $\phi=0$ at the wall and the peak value $\phi_{\max }$. Similarly, the selection of streamlines correspond to equally spaced values of the stream function $\psi$, defined such that $r v_{r}=\partial \psi / \partial z$ and $r v_{z}=-\partial \psi / \partial r$ with $\psi=0$ at the wall, and the peak values being $\psi_{\max }=$ $\left(1.6822 \times 10^{-3}, 1.5575 \times 10^{-3}, 5.728 \times 10^{-4}, 1.361 \times 10^{-4}, 2.7973 \times\right.$ $\left.10^{-5}, 5.6811 \times 10^{-6}\right)$ for $R a=7 \times\left(0,10^{2}, 10^{3}, 10^{4}, 10^{5}, 10^{6}\right)$.

It can be seen that the Frank-Kamenetskii vortex induced by buoyancy, symmetric about the equatorial plane for $R a \ll 1$, evolves in a non-monotonic manner for increasing $R a$, with the accompanying velocity, directed upwards in the central hot region, causing a vertical displacement of the peak temperature, which readily destroys the spherical symmetry of the temperature distribution, as noticed in early experimental observations $[23,24]$. The streamline pattern indicates that the center of the vortex, which initially migrates upwards, moves eventually downwards to occupy a location near the wall, where a high-velocity boundarylayer flow develops for $R a \gg 1$, as inferred by the close spacing between adjacent streamlines. The structure of the flow in that limit, which includes a central region of stratified temperature with a hot kernel near the "north pole" of the vessel, is to be considered in detail in Section 4.

Unlike numerical schemes based on pseudo-transient methods, the root-finding technique employed here not only is able to describe the lower branch of stable solutions, extending between the origin of the explosion diagram $\phi_{\max }-D a$ and the turning point of the bending bifurcation, but it can also be employed in principle to describe the unstable solutions lying beyond the turning point, corresponding to larger values of $\phi_{\max }$. For illustrative purposes, a detailed view of the turning point of the curve corresponding to $R a=700$ is shown in Fig. 2, along with the isotherms and streamlines corresponding to two different solutions for the same $D a<D a_{c}$. The description of the solutions along the upper branch becomes more difficult for larger $R a$, because the curvature of the associated explosion curves at the turning point becomes smaller, leading to rapid variations of $\phi_{\max }$ for $D a_{c}-D a \ll 1$ that hinder the convergence of the root-finding scheme. For these larger values of $R a$, the value of $D a_{c}$ reported here is defined as the maximum value 

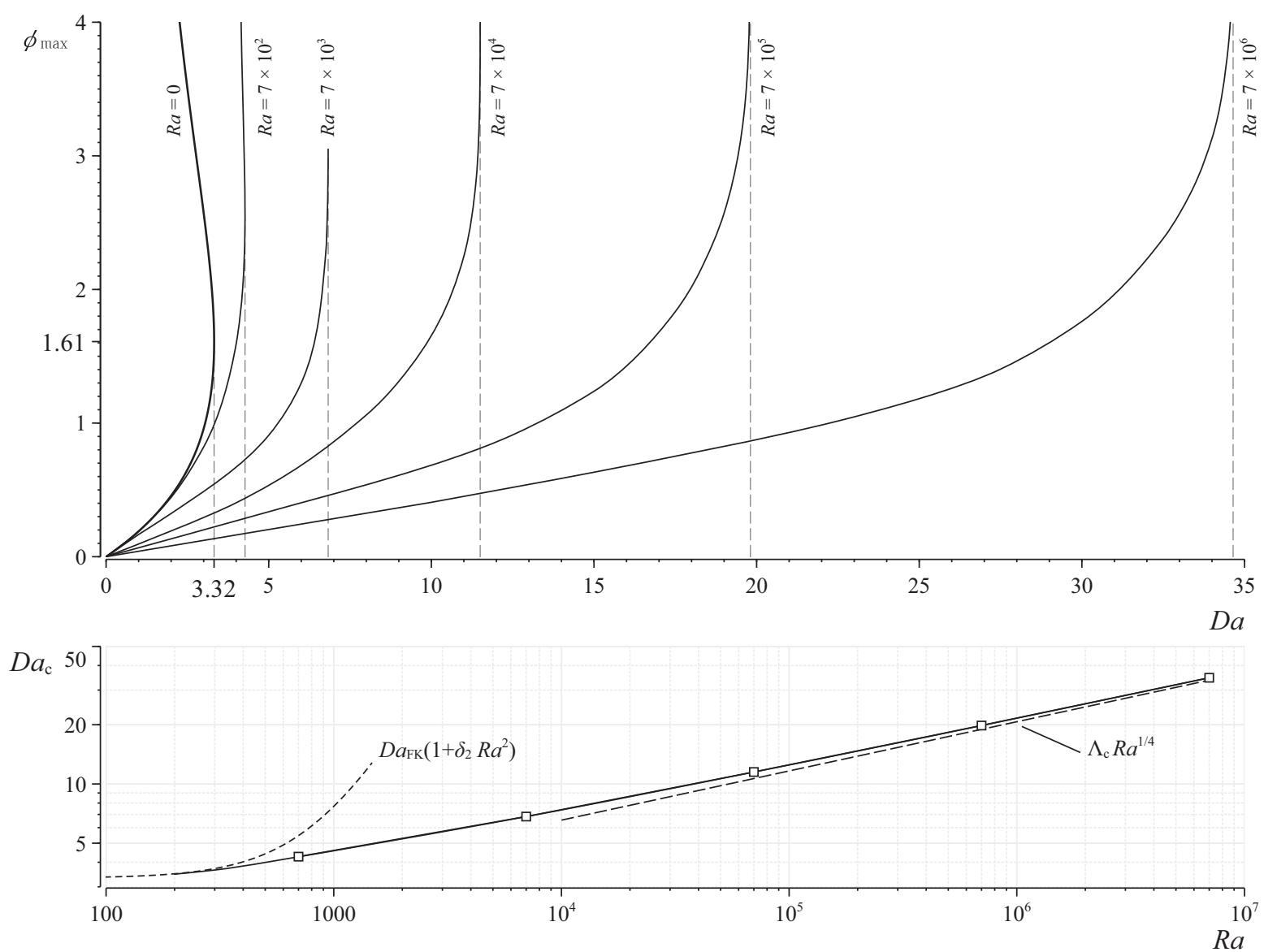

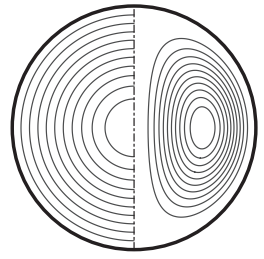

$R a=0$

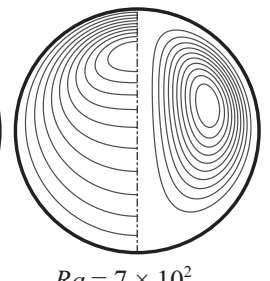

$R a=7 \times 10^{2}$

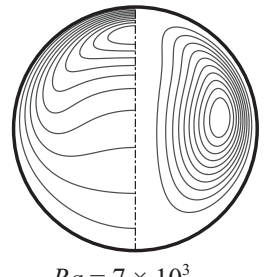

$R a=7 \times 10^{3}$

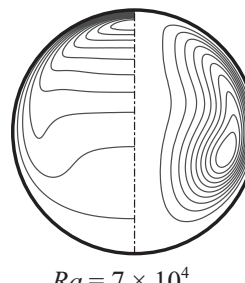

$R a=7 \times 10^{4}$

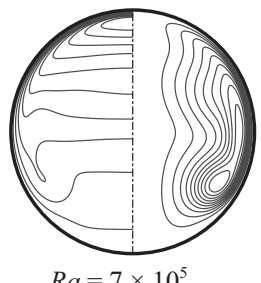

$R a=7 \times 10^{5}$

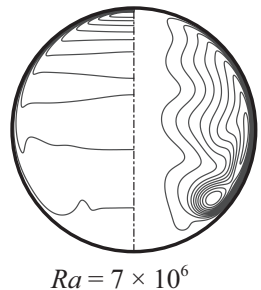

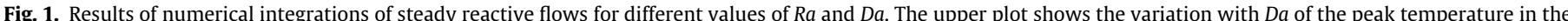

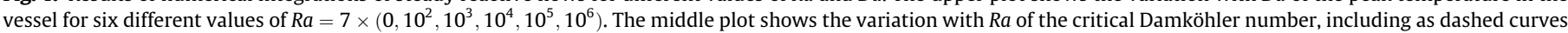

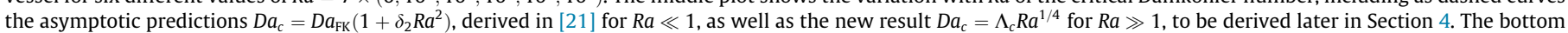
plots show ten isotherms (left half) and ten streamlines (right half) for the conditions corresponding to the six turning points of the upper plot.

of $D a$ for which a converged solution is found. Numerical methods based on continuation techniques might be better suited for the description of the complete multi-branch explosion curve developing beyond the first turning point, but this alternative approach is not further pursued here because of the limited practical interest in these unstable solutions.

\subsection{Transient computations}

To complement the above steady solutions, transient histories corresponding to the initial conditions (11) were computed by marching in time the Eqs. (5)-(7) supplemented with (9), with the boundary conditions given in (10). Besides the parameters $R a$ and $D a$, the Prandtl number $P r=0.7$, and the specific-heat ratio $\gamma=1.4$, the results of the transient computations depend also on $\phi_{I}$, the rescaled initial gas temperature in the container. As previously discussed, to ensure that the chemical reaction is negligible before the wall temperature is suddenly increased at $t=0$, this negative temperature must satisfy $-\phi_{I} \gg 1$. The value $\phi_{I}=-10$ is employed in the computations reported below.

As in the steady computations, finite elements were used for the spatial discretization. The time-marching technique employed a characteristics-Galerkin method [22,25] with a fixed time step $\Delta t$. The resulting system of linearized equations was solved at each time step using FreeFem++ [22]. The temporal evolution, including the associated ignition time for supercritical cases, was found to be independent of the choice of $\Delta t$ provided that a sufficiently small value is employed. The solutions shown below in Figs. 3-5 correspond to $\Delta t=10^{-5}$.

As expected, the transient computations reproduce the FK explosion boundary $D a_{c}(R a)$ shown in the middle plot of Fig. 1 in that, regardless of the value of $R a$, the unsteady flow for all subcritical values of the Damköhler number $D a<D a_{c}(R a)$ was seen to evolve to reach the final steady-state solutions shown in Fig. 1 with indistinguishable differences, whereas for $D a>D a_{c}(R a)$ a localized thermal runaway occurs at a finite time $t=t_{i}$, larger for smaller values of $D a-D a_{c}$. 


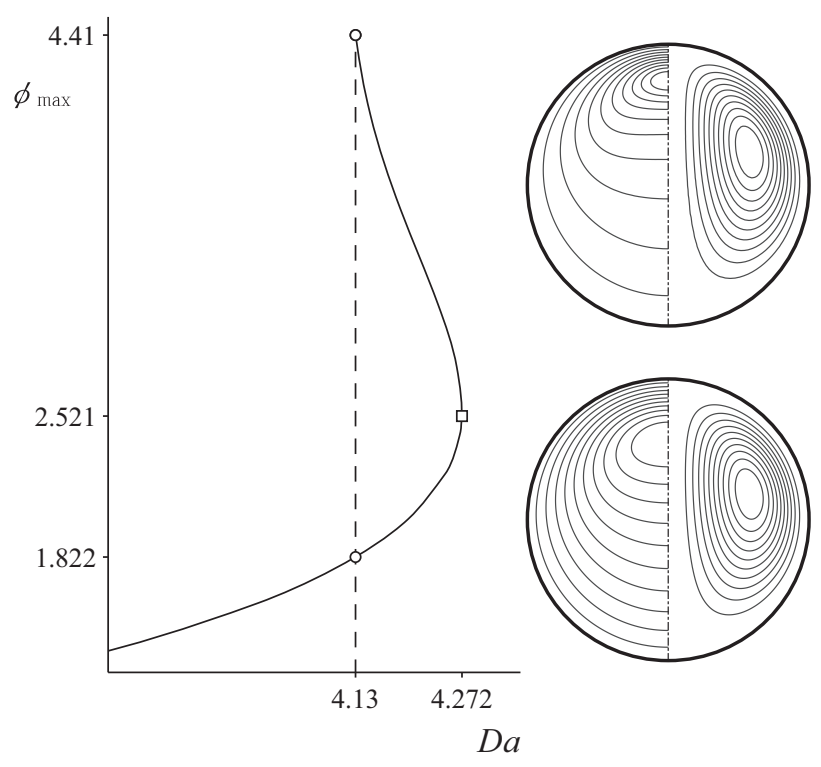

Fig. 2. Detailed view of the explosion curve near the turning point for $R a=700$. The circular plots show isotherms (left half) and streamlines (right half) corresponding to $D a=4.13$ for the solutions along the upper (unstable) branch and the lower (stable) branch. A total of 10 equally spaced isocurves is shown in each plot, with peak values of temperature and stream function given by $\phi_{\max }=4.410$ and $\psi_{\max }=1.594 \times 10^{-3}$ for the upper plot and $\phi_{\max }=1.822$ and $\psi_{\max }=1.380 \times 10^{-3}$ for the lower plot.

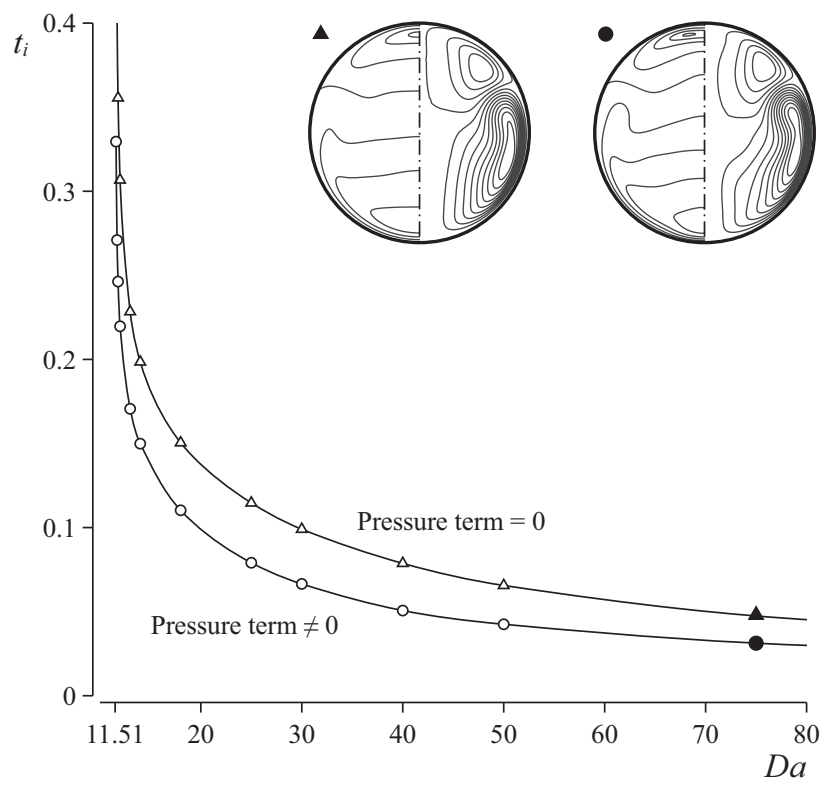

Fig. 3. Ignition time for $R a=7 \times 10^{4}$ as a function of $D a>D a_{c}(R a) \simeq 11.51$. The inset shows isotherms and streamlines for $D a=75$ at $t \approx t_{i}$.

The variation of $t_{i}$ with $D a$ is shown in Fig. 3 for $R a=7 \times 10^{4}$. In the plot, the ignition time is defined as the last time for which a bounded solution is found in the numerical integration. Because of the precipitous character of the temperature increase, other definitions of the ignition time, based for instance on a given threshold value for the local rate of temperature increase, would produce almost identical results. The plot includes the curve $t_{i}(\mathrm{Da})$ obtained by neglecting in the energy Eq. (7) the term involving the temporal pressure increase. In view of these results, it can be concluded that computations of thermal explosions omitting this term, as was done in many previous studies [16,17], may result in significant overpredictions of ignition times in chambers with rigid walls. The plot also indicates that, as expected, the computation of the critical value $D a_{c}$ at which $t_{i} \rightarrow \infty$ is not affected by the presence of this unsteady term. To illustrate changes in the morphology of the ignition kernel, the insets in the figure show snapshots of isotherms and streamlines immediately before ignition for $D a=75$. For this particular case, the ignition kernel is centered at a point along the axis when the term $\mathrm{d} \hat{p} / \mathrm{d} t$ is removed, but ignition occurs in a thin reacting annulus, slightly detached from the container axis, when this term is retained in the computation, as may be seen at the top of the insets.

As previously stated, the results in Fig. 3 correspond to $\phi_{I}=-10$. Since the duration of the heating period needed to increase the gas temperature in the container to values close to the wall value depends fundamentally on $\phi_{I}$, lower values of $\phi_{I}$ result in larger ignition times, as was verified in additional computations. Nevertheless, the explosion limits, measured by the critical Damköhler number $D a_{c}$, were found to be independent of the selection of $\phi_{I}$ for all $\phi_{I} \leqslant 0$, in agreement with our previous results for buoyancy-free systems [5].

The role of buoyancy in the development of the thermal explosion is investigated in Fig. 4 for $R a=7 \times 10^{4}$ and $\phi_{I}=-10$ when the Damköhler number is $D a=11.75$, only slightly above the critical value $D a_{c}=11.51$ predicted by the turning-point FK criterion. The upper left panel shows the temperature at the center of the vessel $\phi_{o}$ and the peak temperature $\phi_{\max }$, the latter displaying a precipitous growth at $t=t_{i} \simeq 0.28$. The twelve panels at the bottom show isotherms and streamlines at representative instants of time, marked with circles in the curve $\phi_{o}(t)$. The shading is used in the panels to indicate positive temperatures (above the wall value) and positive values of the stream function, corresponding to counterclockwise motion in the right-hand-side panels.

The heating of the gas from the hot wall affects initially a thin boundary layer that moves upwards under the action of gravity, entraining the surrounding cold gas and inducing a counterclockwise motion on the right inside the vessel. Because of the spherical geometry of the container, the boundary layer collides at the top of the sphere, ejecting downwards along the axis the gas that has been heated while ascending next to the wall. A secondary vortex with clockwise motion is seen to emerge from the collision region, thereby further complicating the dynamics. This convective transport rapidly heats the upper half of the vessel, while the bottom, nearly stagnant, remains initially cold.

The chemical reaction, initially negligible, begins to occur near the top of the sphere, causing the temperature to increase there above the wall value (i.e. positive values of $\phi$ ) for $t \geq 0.017$. The shaded region representing the hot gas extends rapidly downstream, changing in a fundamental way the gas motion. This can be seen in the snapshot corresponding to $t=0.0643$ (the third from the left on the bottom row). In the upper half of the vessel $\phi>0$, so that in the corresponding near-wall boundary layer the gas temperature is lower than that found outside. Consequently, buoyancy pulls the fluid downwards near the wall, supporting clockwise motion in this region. The opposite effect is found on the lower half of the vessel, where the temperature still remains negative. As a result, the flow at this intermediate time is characterized by the existence of two annular counterrotating vortices. For longer times, the hot gas occupies the entire vessel, and the resulting motion is given by a single vortex with clockwise motion, as in the steady cases displayed in Fig. 1. The change in sign of the heat flux from the wall and the corresponding flow reversal along the boundary layer are illustrated in the upper right plot of Fig. 4 by plotting the radial distributions of vertical velocity and temperature at the equatorial plane $z=0$ at different instants of time. 

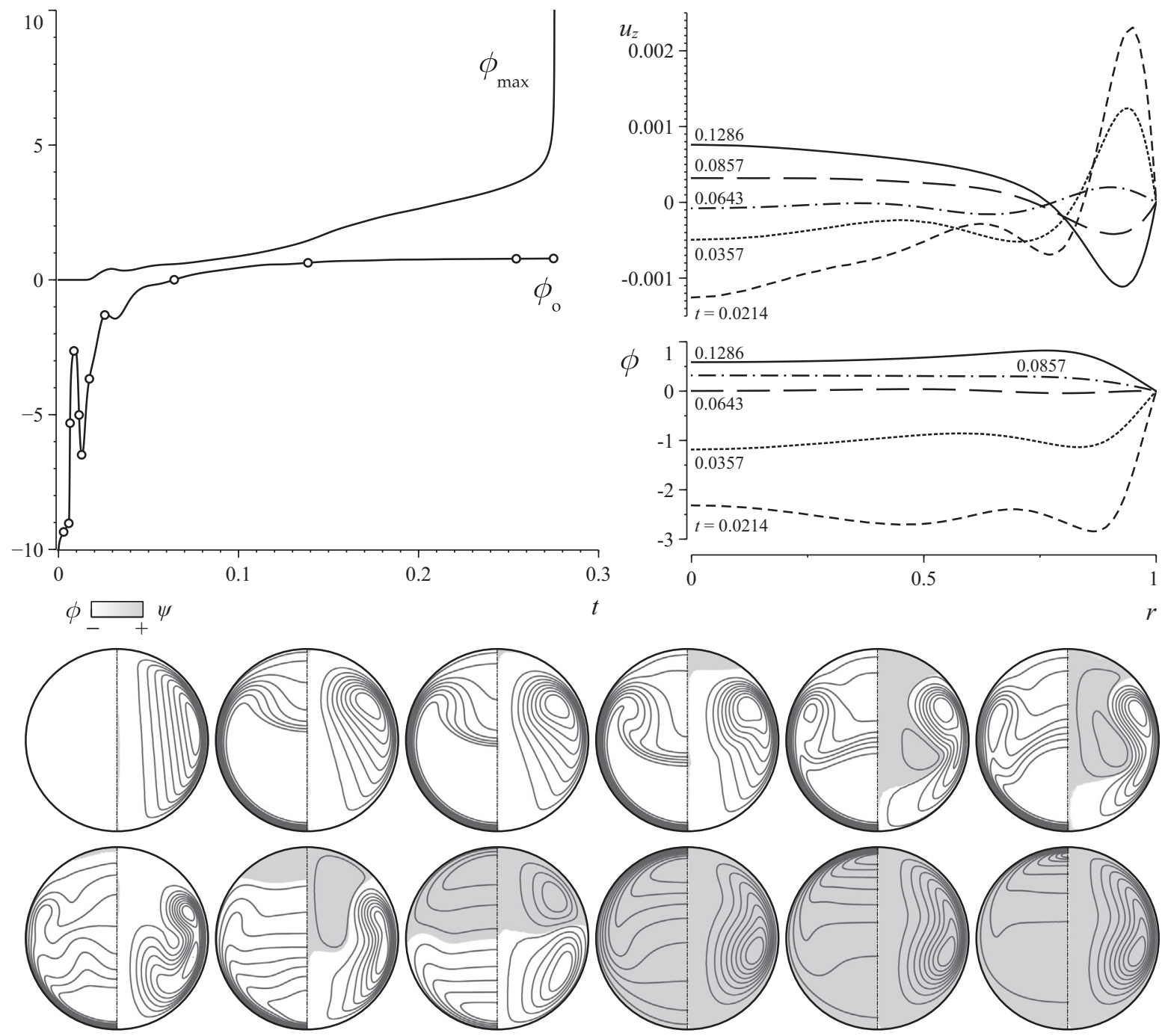

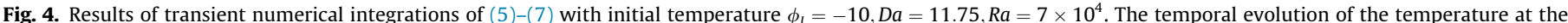

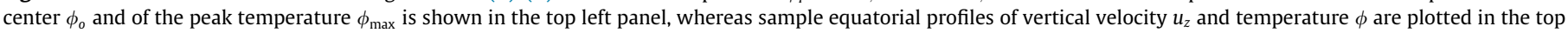

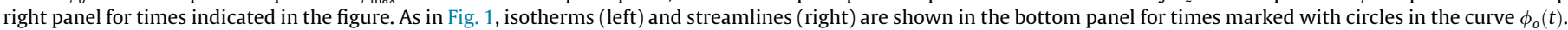

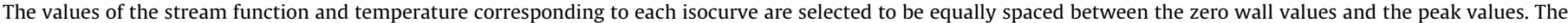
shade indicate positive values of the temperature (left half) and stream function (right half).

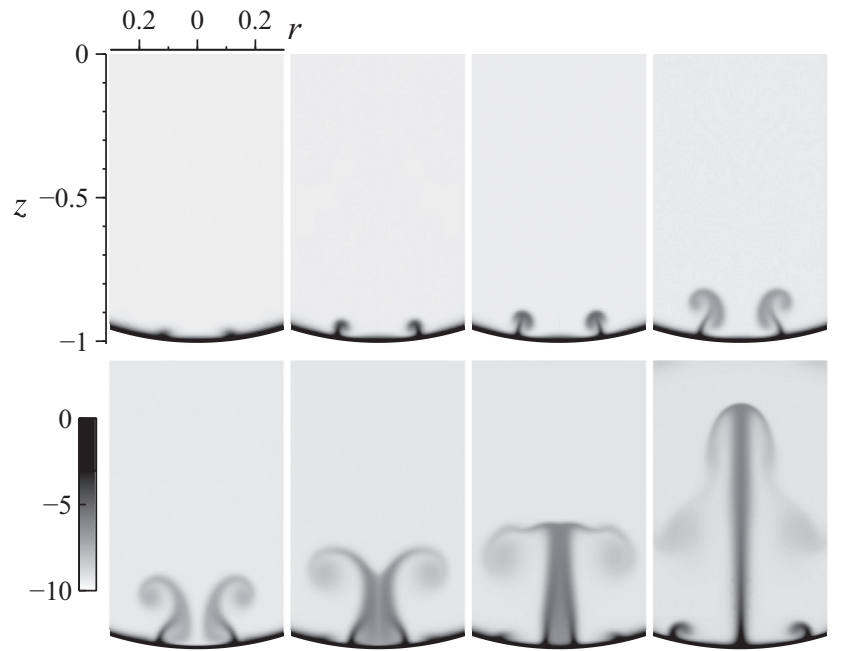

Fig. 5. Snapshots at $t=(0.93,1.07,1.14,1.29,1.43,1.64,1.79,2.00) \times 10^{-3}$ showing the formation of a plume as a result of a thermal instability near the bottom of the vessel for $R a=7 \times 10^{6}$. The shading represents temperature, varying between -10 (light) and 0 (dark).
As seen in the last three snapshots in Fig. 4, corresponding to $t=(0.1386,0.2543,0.2750)$, for this near-critical case the transient ignition history shows a relatively long stage of slow evolution in which the streamline pattern at all times is very similar to that of the FK solution at the turning point (see the plot for $R a=7 \times 10^{4}$ in Fig. 1). This quasi-steady motion is accompanied by a slow temperature evolution in which the values found in the central region remain almost constant, as seen in the evolution of $\phi_{0}$, while the temperature distribution near the top develops an increasingly sharp peak that eventually transitions into a rapid runaway.

The heating dynamics becomes even more complicated for larger values of the Rayleigh numbers because of the development of flow instabilities. In particular, the thin layer of heated gas near the bottom may be subject to Rayleigh-Taylor-like instabilities that lead to the ejection of hot gas. This is illustrated in the series of snapshots shown in Fig. 5, corresponding to the initial stages of the heating process for $R a=7 \times 10^{6}$. It can be seen that the ejection occurs in the form of a toroidal plume, which soon develops into a thermal rising along the axis to eventually meet the jet of fluid coming down from the top collision region. The ejection process repeats itself in a quasi-periodic fashion, thereby providing 
an effective mechanism that contributes to the heating of the vessel interior.

It is worth noting that the dynamics described here is limited by the axisymmetric character of the integrations. The computations are not capable of describing the three-dimensional boundarylayer instabilities that are expected to be responsible for the transition to turbulence that has been postulated to occur for $R a \gtrsim 10^{6}$ $[16,17]$. Uncertainties remain concerning exactly when transition occurs, but it seems likely that the patterns revealed in Fig. 5 will play some role in post-transitional development of the flow.

\section{Slowly reacting flow for $R a \gg 1$}

\subsection{Flow structure}

The computations shown in Fig. 1 reveal that the structure of the flow in the limit $R a \gg 1$ includes a central region of slowly moving hot gas bounded by a high-velocity near-wall boundary layer. The scales and the dominant balances applying in each region can be identified by an order-of-magnitude analysis. Thus, since the temperature increment must be $\phi \sim 1$ for the thermal explosion to develop, the buoyancy force in (6) can be anticipated to be of order unity when $D a \sim D a_{c}$, inducing streamwise velocities of order $R a^{-1 / 2}$ in a near-wall boundary layer of characteristic thickness $R a^{-1 / 4}$, as inferred from the convection-diffusion balance in (6). The flow in the central region, outside the boundary layer, is induced by the boundary-layer entrainment, with characteristic velocities of order $R^{-3 / 4}$. This slow motion does not induce an appreciable pressure disturbance inside the container, where (6) reduces in the first approximation to $-\nabla p^{\prime}+\phi \boldsymbol{e}_{z}=0$. This hydrostatic balance requires that the pressure $p^{\prime}$ and the temperature $\phi$ be a function of $z$ only. This is consistent with the results shown in Fig. 1, where the isotherms for $R a \gg 1$ are nearly horizontal outside the boundary layer. Since the velocities induced by boundarylayer entrainment are of order $R a^{-3 / 4}$, the effective Peclet number, measuring in the central region the relative importance of convective and conductive rates, is of order $R a^{1 / 4} \gg 1$, so that the effect of heat conduction can be neglected in the first approximation when writing (7) there. The resulting convection-reaction balance $R a \boldsymbol{v} \cdot \nabla \phi=R a \mathrm{e}^{\phi}$, with $\boldsymbol{v} \sim R a^{-3 / 4}$, indicates that for $D a \sim 1$ the temperature increase associated with the chemical reaction is limited to small values of order $R a^{-1 / 4}$, insufficient to produce a significant increase in the reaction rate from its near-wall value, and that temperature increments of order unity, needed to trigger the thermal explosion, require values of the Damköhler number of order $R a^{1 / 4}$. In this near-explosion regime, $D a \sim R a^{1 / 4}$, the chemical reaction occurs mainly in the central region, while the boundary layer remains chemically frozen in the first approximation, because the transport rates there are larger than the reaction term by a factor of order $R a^{1 / 4}$, as can be seen from (7).

Before proceeding with the analysis, it is worth pointing out that the flow structure identified here is fundamentally similar to that emerging in fully developed flow in a curved pipe at large values of the Dean number [26]. In both cases, the inviscid internal flow is coupled with a thin boundary layer that develops between two stagnation-point self-similar regions. Also, as we shall see below, the difficulties in the numerical solution stem in both cases from the elliptic character of the boundary-layer problem, associated with the existence of recirculating flow.

\subsection{The boundary-layer problem}

The leading-order form of the simplified equations in the chemically frozen boundary layer and in the central inviscid region are written now in terms of appropriately rescaled variables. The boundary layer developing at the wall is described in terms of the local distance to the wall $y=R a^{1 / 4}\left[1-\left(r^{2}+z^{2}\right)^{1 / 2}\right]$ and the angle $\theta=\cos ^{-1} z$, which measures the distance along the wall from the vessel top, giving the equations

$$
\begin{aligned}
& \frac{\partial}{\partial \theta}(U \sin \theta)+\frac{\partial}{\partial y}(V \sin \theta)=0 \\
& \frac{1}{\operatorname{Pr}}\left(U \frac{\partial U}{\partial \theta}+V \frac{\partial U}{\partial y}\right)=\frac{\partial^{2} U}{\partial y^{2}}+\left(\phi_{c}-\phi\right) \sin (\theta) \\
& U \frac{\partial \phi}{\partial \theta}+V \frac{\partial \phi}{\partial y}=\frac{\partial^{2} \phi}{\partial y^{2}}
\end{aligned}
$$

where $U=R a^{1 / 2}\left(z v_{r}-r v_{z}\right)$ and $V=-R a^{3 / 4}\left(r v_{r}+z v_{z}\right)$ are the rescaled streamwise and transverse velocity components, respectively. The boundary conditions for $0<\theta<\pi$ are

$$
\begin{aligned}
y=0: & U=V=\phi=0 \\
y \rightarrow \infty: & U=\phi-\phi_{c}(\theta)=0 .
\end{aligned}
$$

Here $\phi_{c}(\theta)$ is the temperature outside the boundary layer, in the central inviscid region, given by the convection-reaction balance

$\bar{v}_{z} \frac{\mathrm{d} \phi_{c}}{\mathrm{~d} z}=\Lambda e^{\phi_{c}}$

where $\Lambda=R a^{-1 / 4} D a$ is a rescaled Damköhler number and $\bar{v}_{z}=R a^{3 / 4} v_{z}$ is the rescaled vertical velocity, also a function of $z$, as required for consistency.

The conduction-free Eq. (17) describes the increase of the temperature of the ascending gas due to chemical reaction, from the value $\phi_{c}=0$, found near the bottom $z=-1$, to reach the peak value $\phi_{\max }$ as $z \rightarrow 1$. The vertical velocity appearing as a factor in (17) can be obtained at a given height $z$ by equating the upward mass flux $\pi\left(1-z^{2}\right) \bar{v}_{z}$ in the container interior to the downward mass flux $2 \pi\left(1-z^{2}\right)^{1 / 2} \int_{0}^{\infty} U \mathrm{~d} y$ across the boundary layer to give

$\bar{v}_{z}=\frac{2 \int_{0}^{\infty} U \mathrm{~d} y}{\left(1-z^{2}\right)^{1 / 2}}$,

and the accompanying radial velocity $\bar{v}_{r}=R a^{3 / 4} v_{r}$ is given by $\bar{v}_{r}=-(r / 2) \mathrm{d} \bar{v}_{z} / \mathrm{d} z$, as follows from continuity. The geometrical relations $\sin \theta=\left(1-z^{2}\right)^{1 / 2}$ and $\sin (\theta) \mathrm{d} \theta=-\mathrm{d} z$ can be used together with (18) to express (17) in the form

$\frac{\mathrm{d} \phi_{c}}{\mathrm{~d} \theta}=-\frac{\Lambda \sin ^{2}(\theta) e^{\phi_{c}}}{2 \int_{0}^{\infty} U \mathrm{~d} y}$,

yielding the solution

$\phi_{c}(\theta)=\ln \left[e^{-\phi_{\max }}+\frac{\Lambda}{2} \int_{0}^{\theta} \frac{\sin ^{2}(\tilde{\theta})}{\int_{0}^{\infty} U \mathrm{~d} y} \mathrm{~d} \tilde{\theta}\right]^{-1}$,

upon integration with the initial condition $\phi_{c}(0)=\phi_{\max }$.

The integration of (12)-(14) must be initiated at $\theta \ll 1$, where there exists a self-similar solution. At the leading order described here, the solution is identical to that encountered near the attachment point of the buoyancy-driven boundary layer that develops over a hot sphere in a stagnant atmosphere when the Grashof number is large [27]. The local flow, first described for the hot sphere in [28], can be analyzed by introducing the normalized coordinate $\zeta=\phi_{\max }^{1 / 4} y$ together with the stream function $\psi=\phi_{\max }^{1 / 4} \theta^{2} F(\zeta)$ and the reduced temperature $G=\left(\phi_{\max }-\phi\right) / \phi_{\max }$ to give

$F_{\zeta \zeta \zeta}+\left(2 F F_{\zeta \zeta}-F_{\zeta}^{2}\right) / P r+G=0$,

$G_{\zeta \zeta}+2 F G_{\zeta}=0$ 
with boundary conditions

$F(0)=F_{\zeta}(0)=G(0)-1=0$

$F_{\zeta}(\infty)=G(\infty)=0$,

where the subscript $\zeta$ denotes differentiation with respect to this coordinate. The integration of (21)-(24) for $P r=0.7$ provides the profiles shown in Fig. 6 along with the value of $F(\infty)=0.63702$, which can be used to write the relationship $\bar{v}_{z}(z=1)=$ $2 \phi_{\max }^{1 / 4} F(\infty)$ between the boundary temperature and the uniform entrainment velocity at $\theta=0$. The uniformity of the boundarylayer entrainment rate near the top is consistent with the negligible radial variation of the vertical velocity $\bar{v}_{z}(z)$ in the reactive core region. The functions $F(\zeta)$ and $G(\zeta)$ provide the profiles of streamwise velocity and temperature

$U=\phi_{\max }^{1 / 2} \theta F_{\zeta} \quad$ and $\quad \phi=\phi_{\max }(1-G) \quad$ at $\theta \ll 1$,

to be used as initial condition for integration of (12)-(14).

The apparent parabolic nature of (13) and (14) suggests a marching procedure in which (12)-(14) supplemented with (20) are integrated for increasing $\theta$ subject to the boundary conditions (16). The integration must be initiated at $\theta \ll 1$ using the selfsimilar profiles (25). In this scheme, the value of $\Lambda$ corresponding to a given maximum temperature $\phi_{\max }$ is obtained from (20) as an eigenvalue by imposing the condition that $\phi_{c} \rightarrow 0$ as $\theta \rightarrow \pi$. This eigenvalue problem bears similarity with that encountered in the analysis of fully developed flow in curved pipes at large Dean number [26]. As in that case, the time-marching integration strategy delineated above fails because of the appearance of recirculating flow. This is observed to emerge at an intermediate location between $\theta=\pi / 2$ and $\theta=\pi$, with the velocity profile $U$ exhibiting regions of negative $U$. This behavior seems to be consistent with the streamline patterns depicted in Fig. 1 for $R a \gg 1$.

Because of the presence of backflow, the character of the resulting boundary-layer problem changes from parabolic to elliptic. The
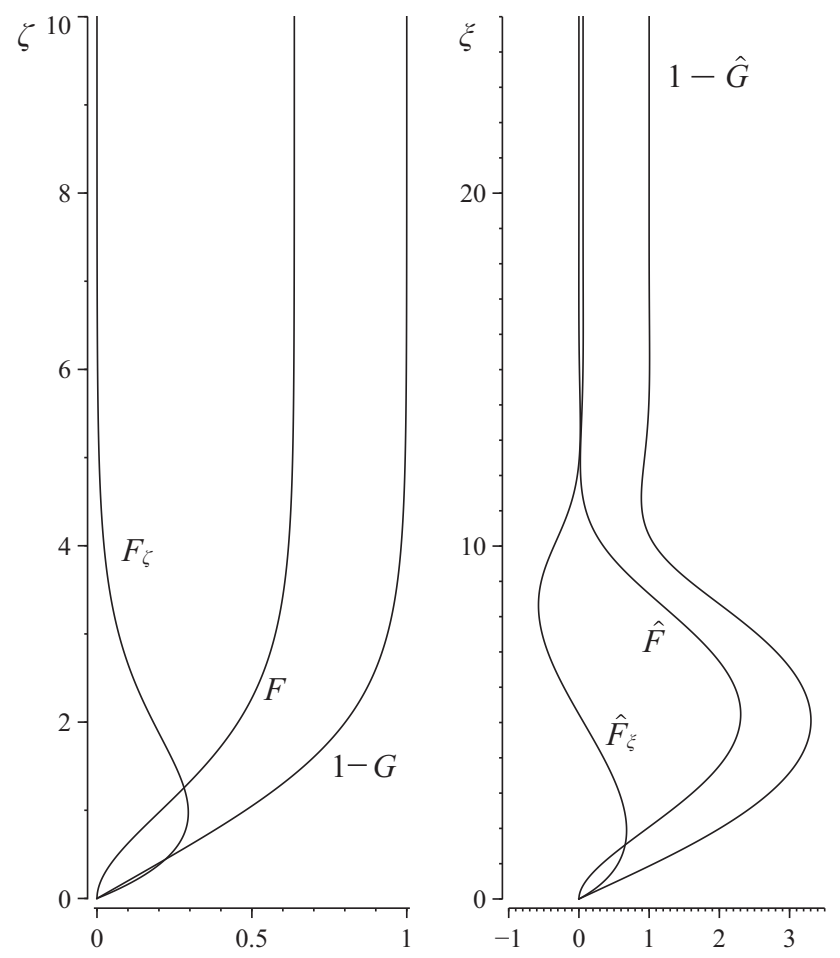

Fig. 6. Results of the integration of (21)-(24) (left panel) and of (26)-(29) (right panel), showing the functions $F(\zeta), F \prime(\zeta), 1-G(\zeta)$ and $\widehat{F}(\xi), \widehat{F}^{\prime}(\xi), 1-\widehat{G}(\xi)$, respectively. The integrations indicate that $F(\infty)=0.63702$ and $\widehat{F}(\infty)=0.05903$. associated boundary-value problem requires consideration of the specific conditions found near the bottom $\theta=\pi$. There, the flow exhibits a self-similar solution, with the core temperature vanishing on approaching $\theta=\pi$ according to the power law $\phi_{c}=A(\pi-\theta)^{\lambda}$, consistent with the local scalings $U \propto(\pi-\theta)^{1+\lambda / 2}$ and $y \propto(\pi-s)^{-\lambda / 4}$, where the value of the exponent $\lambda=8 / 5$ is determined from the convective-reactive balance (19). Writing (12)-(14) in terms of the local coordinate $\xi=A^{1 / 4}(\pi-\theta)^{2 / 5} y$, stream function $\psi=A^{1 / 4}(\pi-\theta)^{12 / 5} \widehat{F}(\xi)$, and temperature increment $\phi_{c}-\phi=A(\pi-\theta)^{8 / 5} \widehat{G}(\xi)$ yields

$\widehat{F}_{\xi \xi \xi}+\left(\frac{9}{5} \widehat{F}_{\xi}^{2}-\frac{12}{5} \widehat{F}_{\xi \xi \xi}\right) / \operatorname{Pr}+\widehat{G}=0$

$\widehat{G}_{\xi \xi}-\frac{12}{5} \widehat{F} \widehat{G}_{\xi}-\frac{8}{5} \widehat{F}_{\xi}(1-\widehat{G})=0$

with boundary conditions

$\widehat{F}(0)=\widehat{F}_{\xi}(0)=\widehat{G}(0)-1=0$

$\widehat{F}_{\xi}(\infty)=\widehat{G}(\infty)=0$,

where the subscript $\xi$ denotes differentiation with respect to this coordinate. The profiles $\widehat{F}, \widehat{F}_{\xi}$, and $\widehat{G}$ are shown in Fig. 6 for $\mathrm{Pr}=0.7$. In connection with this self-similar solution it is worth noting that the boundary-layer thickness diverges as $(\pi-\theta)^{-2 / 5}$ as $\theta \rightarrow \pi$, indicative of a boundary layer that empties near the bottom, a flow feature consistent with the streamline patterns observed in the bottom plots of Fig. 1 for $R a \gg 1$. This type of local behavior is fundamentally different from that emerging in free convection from a heated sphere, where the eruption of the fluid into the plume above the sphere is the result of the collision of the boundary layer at the upper stagnation point, as described by Potter and Riley [27]. Also of interest is that the rescaled streamwise velocity $\widehat{F}_{\xi}$ shown in Fig. 6 displays an oscillatory profile with multiple zeros. An emptying boundary layer with oscillatory velocity profile is also encountered near the inside bend of curved pipes at large Dean number [29], thereby further highlighting the similarities between both flows. The self-similar profiles can be used to write the velocity and temperature distributions as

$$
\begin{aligned}
U & =A^{1 / 2}(\pi-\theta)^{9 / 5} \widehat{F}_{\xi}, \quad \phi_{c}-\phi=A(\pi-\theta)^{8 / 5} \widehat{G} \quad \text { at } \quad \pi-\theta \\
& \ll 1,
\end{aligned}
$$

where the constant factor $A=[(5 \Lambda) /(16 \widehat{F}(\infty))]^{4 / 5}$, obtained from (19), can be evaluated with use made of the boundary value $\widehat{F}(\infty)=0.05903$ determined from the integration of (26)-(29).

Eqs. (12)-(14) must be integrated for $0<\theta<\pi$ together with (20) subject to the boundary conditions (16) and to the local boundary distributions (25) and (30) at $\theta \ll 1$ and $\pi-\theta \ll 1$, respectively. In the solution, the value of $\Lambda$ corresponding to a given $\phi_{\max }$ is an eigenvalue of the problem. The numerical solution of the above boundary-value problem was attempted using different methods, involving either a pseudo-transient scheme for advancing (12)-(14) or the simultaneous solution for $U, V$, and $\phi$ by means of a Newton-Raphson method, with the eigenvalue $\Lambda$ updated after each iteration using (20). Despite significant efforts, difficulties associated with the strong sensitivity of the results to small changes in $\Lambda$ precluded convergence of the method, so that a numerical solution to the problem could not be achieved. It is worth pointing out that similar difficulties have been encountered in connection with the boundary-value problem arising in curved pipes at large Dean number [26], whose numerical solution also remains elusive to date. For this latter problem, an approximate solution based on the integral form of the boundary-layer equations was successfully employed by Ito [30]. The same strategy is 
followed below to obtain the reduced explosion curve $\phi_{\max }$ as a function of $\Lambda$ and the associated critical value $\Lambda_{c}$.

\subsection{Von-Karman integral formulation}

The approximate solution begins by integrating (13) and (14) across the boundary layer to give the two ordinary differential equations

$$
\begin{aligned}
& \frac{1}{\operatorname{Pr}} \frac{\mathrm{d}}{\mathrm{d} \theta}\left(\sin \theta \int_{0}^{\infty} U^{2} \mathrm{~d} y\right)=-\left.\sin \theta \frac{\partial U}{\partial y}\right|_{y=0}+\sin ^{2} \theta \int_{0}^{\infty}\left(\phi_{c}\right. \\
&-\phi) \mathrm{d} y, \\
& \frac{\mathrm{d}}{\mathrm{d} \theta}\left(\sin \theta \int_{0}^{\infty} U\left(\phi_{c}-\phi\right) \mathrm{d} y\right)-\sin \theta \frac{\mathrm{d} \phi_{c}}{\mathrm{~d} \theta} \int_{0}^{\infty} U \mathrm{~d} y=\left.\sin \theta \frac{\partial \phi}{\partial y}\right|_{y=0} .
\end{aligned}
$$

Guided by the method employed in [30], it is further assumed that the boundary layer has a finite thickness $\delta(\theta)$ and that the temperature and streamwise velocity across the boundary layer can be expressed in the form

$\phi=\phi_{c} k(\eta)$ and $U=\phi_{c} \sin (\theta) \delta^{2} f(\eta)+U_{o} h(\eta)$,

where the description for $U$ incorporates a velocity correction $U_{0}(\theta)$ in addition to the direct effect of the gravitational acceleration. The functions $k(\eta), f(\eta)$, and $h(\eta)$ of the normalized transverse coordinate $\eta=y / \delta$ are introduced to define the shape of the profiles. These shape functions are defined such that $k-1=f=h=0$ for $\eta \geqslant 1$, with polynomial expressions employed below for describing $k(\eta), f(\eta)$, and $h(\eta)$ in the range $0 \leqslant \eta \leqslant 1$. In principle, a secondorder polynomial for $k$ and $f$ and a third-order polynomial for $h$ suffice to satisfy the boundary conditions

$$
\left\{\begin{array}{l}
k=f=h=0 \text { and } \quad \frac{\mathrm{d}^{2} k}{\mathrm{~d} \eta^{2}}=\frac{\mathrm{d}^{2} f}{\mathrm{~d} \eta^{2}}+1=\frac{\mathrm{d}^{2} h}{\mathrm{~d} \eta^{2}}=0 \text { at } \eta=0 \\
k-1=f=h=0 \quad \text { at } \eta=1,
\end{array}\right.
$$

stemming from (16). Smoother profiles with vanishing derivatives at $\eta=1$ can be obtained by increasing the order of the polynomials. In particular, the results shown below correspond to

$k=\eta\left(2-2 \eta^{2}+\eta^{3}\right), \quad f=\frac{1}{6} \eta(1-\eta)^{3}, \quad h=\eta(1+3 \eta)(1-\eta)^{3}$

which satisfy the additional boundary conditions

$\frac{\mathrm{d} k}{\mathrm{~d} \eta}=\frac{\mathrm{d} f}{\mathrm{~d} \eta}=\frac{\mathrm{d} h}{\mathrm{~d} \eta}=\frac{\mathrm{d}^{2} k}{\mathrm{~d} \eta^{2}}=\frac{\mathrm{d}^{2} f}{\mathrm{~d} \eta^{2}}=\frac{\mathrm{d}^{2} h}{\mathrm{~d} \eta^{2}}=0 \quad$ at $\quad \eta=1$.

The temperature in the interior of the vessel $\phi_{c}(\theta)$, the velocity correction $U_{o}(\theta)$, and the boundary-layer thickness $\delta(\theta)$ are to be computed by integration of (19) written in the form

$\frac{\mathrm{d} \phi_{c}}{\mathrm{~d} \theta}=-\frac{\Lambda \sin ^{2}(\theta) e^{\phi_{c}}}{2 \delta\left(\phi_{c} \sin (\theta) \delta^{2} \int_{0}^{1} f \mathrm{~d} \eta+U_{o} \int_{0}^{1} h \mathrm{~d} \eta\right)}$,

together with the momentum and energy Eqs. (31) and (32) written with use made of the expressions

$$
\begin{gathered}
\int_{0}^{\infty} U^{2} \mathrm{~d} y=\phi_{c}^{2} \sin ^{2}(\theta) \delta^{5} \int_{0}^{1} f^{2} \mathrm{~d} \eta+2 U_{o} \phi_{c} \sin (\theta) \delta^{3} \int_{0}^{1} f h \mathrm{~d} \eta \\
+U_{o}^{2} \delta \int_{0}^{1} h^{2} \mathrm{~d} \eta \\
\int_{0}^{\infty} U\left(\phi_{c}-\phi\right) \mathrm{d} y=\phi_{c}^{2} \sin (\theta) \delta^{3} \int_{0}^{1} f(1-k) \mathrm{d} \eta \\
+U_{o} \phi_{c} \delta \int_{0}^{1} h(1-k) \mathrm{d} \eta
\end{gathered}
$$

$$
\begin{aligned}
& \int_{0}^{\infty}\left(\phi_{c}-\phi\right) \mathrm{d} y=\phi_{c} \delta \int_{0}^{1}(1-k) \mathrm{d} \eta \\
& \int_{0}^{\infty} U \mathrm{~d} y=\phi_{c} \sin (\theta) \delta^{3} \int_{0}^{1} f \mathrm{~d} \eta+U_{o} \delta \int_{0}^{1} h \mathrm{~d} \eta \\
& \left.\frac{\partial U}{\partial y}\right|_{y=0}=\left.\phi_{c} \sin (\theta) \delta \frac{\mathrm{d} f}{\mathrm{~d} \eta}\right|_{\eta=0}+\left.\frac{U_{o}}{\delta} \frac{\mathrm{d} h}{\mathrm{~d} \eta}\right|_{\eta=0} \\
& \left.\frac{\partial \phi}{\partial y}\right|_{y=0}=\left.\frac{\phi_{c}}{\delta} \frac{\mathrm{d} k}{\mathrm{~d} \eta}\right|_{\eta=0} .
\end{aligned}
$$

The equations were integrated by marching in $\theta$. The integration is started with the initial values

$\phi=\phi_{\max }, \quad U_{o}=C_{U} \phi_{\max }^{1 / 2} \theta, \quad \delta=C_{\delta} \phi_{\max }^{-1 / 4} \quad$ at $\quad \theta \ll 1$,

consistent with the previously identified self-similar solution (25). The constant factors $C_{U}$ and $C_{\delta}$ are determined from the system of algebraic equations obtained by substituting (44) into (31) and (32), yielding the values $C_{U}=0.021683$ and $C_{\delta}=4.05827$ for the shape functions (35). The condition $\phi_{c}(\pi)=0$ is used to determine the value of $\Lambda$ corresponding to a given value of $\phi_{\max }$, producing the results shown in Fig. 7.

The lower plot in Fig. 7 represents typical profiles of $\delta, U_{o}$, and $\phi_{c}$ for a large value of $\phi_{\max }$ (e.g., $\phi_{\max }=7$ in the computation shown in the figure). Although the model (33) allows for the presence of backflow in the boundary layer, as would occur if $U_{o}$ reaches sufficiently large negative values, such recirculating flow conditions were not achieved for any $\phi_{\max }$; instead, $U_{o}$ was seen to remain always positive for $0 \leqslant \theta \leqslant \pi$. The nonzero value of $U_{o}$ as $\theta \rightarrow \pi$, indicative of boundary-layer collision, can be attributed to the inherent limitations of this approximate model. As in Ito's analysis [30], the thickness of the mixing layer $\delta(\theta)$ shows a nonmonotonic behavior for increasing $\theta$ and eventually diverges as $\theta \rightarrow \pi$, the latter boundary-layer thickening being consistent with
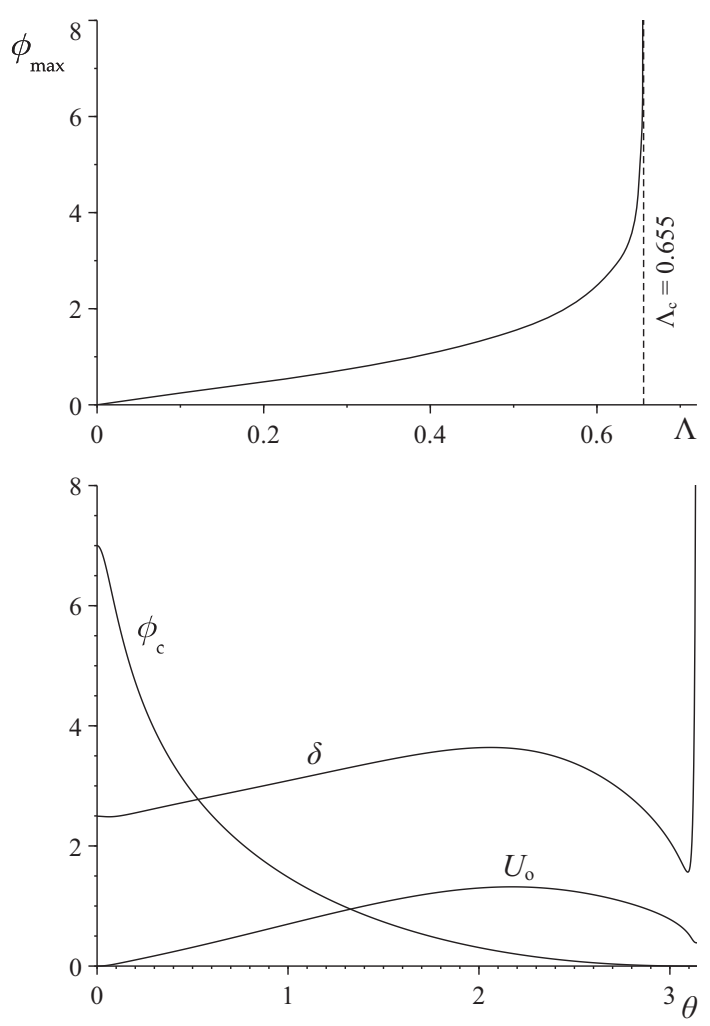

Fig. 7. Results of numerical integrations of (31), (32) and (37) for the shape functions given in (35). 
the self-similar scaling $\delta \sim(\pi-\theta)^{-2 / 5}$ identified earlier for $\pi-\theta \ll 1$.

The upper plot in Fig. 7 shows the reduced explosion curve (i.e., the variation of the reduced Damköhler number $\Lambda$ with $\phi_{\max }$ ). The integral method is not capable of describing the bending bifurcation; it yields instead a value of $\Lambda$ that approaches a limiting value $\Lambda=\Lambda_{c}$ as $\phi_{\max } \rightarrow \infty$, seen in the upper plot. The vertical asymptote, rather than the turning point, identifies in this case the explosion limit, thereby providing the leading-order prediction

$D a_{c}=\Lambda_{c} R a^{1 / 4}$.

For the polynomials (35) used in Fig. 7 the critical Damköhler number is found to correspond to $\Lambda_{c} \simeq 0.655$. Although a different selection of shape functions $k(\eta), f(\eta)$, and $h(\eta)$ results in a different value of $\Lambda_{c}$, the associated changes were verified in additional computations to be only moderate. For instance, use of third-order polynomials for $k$ and $f$ and a fourth-order polynomial for $h$ (satisfying (34) and having vanishing first-order derivatives at $\eta=1$ ) yields instead the value $\Lambda_{c}=0.616$. Since (45) can be expected to have relative errors of order $\mathrm{Ra}^{-1 / 4}$ (that being the order of magnitude of the terms left out in the leading-order asymptotic description), the differences in $\Lambda_{c}$, on the order of $5 \%$, are not very important for practical purposes, in that up to large values of $R a \sim 10^{6}$ they lead to differences in predictions of $D a_{c}$ that are comparable to or smaller than the inherent errors of the leadingorder result (45).

The asymptotic prediction (45) evaluated with $\Lambda_{c}=0.655$ is compared in the middle plot of Fig. 1 with the critical Damköhler numbers computed numerically from integration of (5)-(7). As can be seen, the accuracy of the prediction is truly remarkable. The relative departures between the two curves remain of order $R a^{-1 / 4}$, consistent with the relative errors present in the leadingorder development leading to (45). Achievement of this degree of success in numerical accuracy with a von-Karman integral method is perhaps somewhat surprising.

\section{Concluding remarks}

Simplified equations, derived for the slowly reacting mode of combustion and accounting for buoyancy-induced motion as well as for the time variations of the pressure in the confined environment of the vessel, provide a useful basis for developing insights into fluid dynamics and heat-transfer mechanisms associated with this ignition process. Numerical integrations of the transient problem successfully describe the development of the thermal explosion. The integrations indicate that, despite the complex dynamics arising during the transient stage, the predictions of explosion limits derived from Frank-Kamenetskii's criterion (i.e., based on the existence of steady solutions with negligible fuel consumption) remain accurate over the whole range of Rayleigh numbers explored in the paper.

In the limit $R a \gg 1$ appropriate scalings can be identified for deriving predictions of critical explosion conditions when the flow is dominated by buoyancy. A characteristic structure emerges, including an inviscid convective-reactive core of slowly rising hot fluid, surrounded by a descending near-wall boundary-layer flow driven by the negative buoyancy associated with the cooler walls. The presence of backflow in the boundary layer gives this boundary-layer problem an elliptic character, resulting in an appreciably complicated boundary-value problem which needs results of analyses of special, simpler boundary layers at the top and bottom of the vessel for determining the appropriate boundary condition for the computation of the boundary layer along the curved walls. An approximate solution method, employing an inte- gral form of the boundary-layer equations, combined with presumed shapes for the temperature and velocity profiles, circumvents computational difficulties that are encountered in attempts to integrate numerically the elliptic boundary-value problem that arises for the boundary layer on the curved walls. The most significant result of the asymptotic analysis for $R a \gg 1$ is the leading-order prediction (45) of the critical Damköhler number for explosion as a function of the Rayleigh number. The value of the proportionality factor $\Lambda_{c} \simeq 0.655$, obtained from the approximate analysis appears to be surprisingly accurate, as may be seen in the comparisons of critical explosion conditions shown in the middle plot of Fig. 1.

This work serves to extend the Frank-Kamentskii theory of thermal explosions of ideal gas mixtures in rigid spherical vessels throughout the entire range of conditions over which buoyancy produces interior flows that remain laminar. The associated fluidflow influences on explosion conditions become important only for Rayleigh numbers above a few hundred, and transitions to turbulent flow occur at Rayleigh numbers of a few million, whence the current results may be useful over about four orders of magnitude of the Rayleigh number. Both numerical and conceptual challenges were encountered, but the correct scalings for laminar flows at large Rayleigh numbers were established, along with the determination of reasonably accurate values for associated constants that appear in the scaling relations. Future studies for large Rayleigh numbers may address peculiar geometrical effects that are known to be encountered in vessels of different shapes (as indicated in the introduction), or turbulent flows at higher Rayleigh numbers.

\section{Conflict of interest}

The authors declare that there is no conflict of interest.

\section{Acknowledgments}

We would like to thank Norman Riley for his numerous enlightening comments regarding our work. In particular, he pointed out the similarities between the boundary-layer problem identified here in the limit $R a \gg 1$ and that emerging in fully developed flow in curved pipes at large values of the Dean number, and he also suggested the use of the integral formulation to obtain an approximate solution. The work of FAW is supported by the US National Science Foundation through award \#CBET-1404026.

\section{References}

[1] D. Frank-Kamenetskii, The temperature distribution in a reaction vessel and the time-independent theory of thermal explosions, Zh. Fiz. Khim. 13 (1939) $738-755$.

[2] D.A. Frank-Kamenetskii, Diffusion and Heat Exchange in Chemical Kinetics, Princeton University Press, 1955.

[3] A.L. Sánchez, F.A. Williams, Recent advances in understanding of flammability characteristics of hydrogen, Prog. Energy Combust. Sci. 41 (2014) 1-55.

[4] A.L. Sánchez, E. Fernández-Tarrazo, F.A. Williams, The chemistry involved in the third explosion limit of $\mathrm{H}_{2}-\mathrm{O}_{2}$ mixtures, Combust. Flame 161 (2014) $111-$ 117.

[5] A. Liñán, D. Moreno-Boza, I. Iglesias, A.L. Sánchez, F.A. Williams, The slowly reacting mode of combustion of gaseous mixtures in spherical vessels. Part 1 : Transient analysis and explosion limits, Combust. Theory Modell. 20 (2016) 1010-1028.

[6] D. Jones, The dynamic stability of confined, exothermically reacting fluids, Int. J. Heat Mass Transf. 16 (1973) 157-167.

[7] A. Kolesnikov, Convective stability of the horizontal reacting liquid layer in the presence of various complicating factors, Int. J. Heat Mass Transf. 35 (1992) 1091-1102.

[8] G. Joulin, A. Mikishev, G. Sivashinsky, A Semenov-Rayleigh-Benard problem Unpublished, 1996.

[9] L. Kagan, H. Berestycki, G. Joulin, G. Sivashinsky, The effect of stirring on the limits of thermal explosion, Combust. Theory Modell. 1 (1997) 97-111. 
[10] E. Shtessel', K. Pribytkova, A. Merzhanov, Numerical solution of the problem of a thermal explosion taking account of free convection, Combust. Explos. Shock Waves 7 (1971) 137-146.

[11] A. Merzhanov, E. Shtessel', Free convection and thermal explosion in reactive systems, Acta Astronaut. 18 (1973) 191-199.

[12] M. Massot, S. Génieys, T. Dumont, V. Volpert, Interaction of thermal explosion and natural convection: critical conditions and new oscillating regimes, SIAM J. Appl. Math. 63 (2002) 351-372.

[13] M. Belk, V. Volpert, Modeling of heat explosion with convection, Chaos: Interdiscipl. J. Nonlin. Sci. 14 (2004) 263-273.

[14] D. Jones, Convective effects in enclosed, exothermically reacting gases, Int. J. Heat Mass Transf. 17 (1974) 11-21.

[15] A. Campbell, S. Cardoso, A. Hayhurst, A comparison of measured temperatures with those calculated numerically and analytically for an exothermic chemical reaction inside a spherical batch reactor with natural convection, Chem. Eng. Sci. 62 (2007) 3068-3082.

[16] T.-Y. Liu, A.N. Campbell, S.S. Cardoso, A.N. Hayhurst, Effects of natura convection on thermal explosion in a closed vessel, Phys. Chem. Chem. Phys. 10 (2008) 5521-5530.

[17] T.-Y. Liu, A.N. Campbell, A.N. Hayhurst, S.S. Cardoso, On the occurrence of thermal explosion in a reacting gas: the effects of natural convection and consumption of reactant, Combust. Flame 157 (2010) 230-239.

[18] W. Archer, Heat Transfer Mechanisms in Exothermic Reactions, Ph.D. thesis, The University of Manchester, 1977.

[19] T.-Y. Liu, S.S. Cardoso, Effects of combined natural and forced convection on thermal explosion in a spherical reactor, Combust. Flame 160 (2013) 191-203.
[20] A. Campbell, The effect of external heat transfer on thermal explosion in a spherical vessel with natural convection, Phys. Chem. Chem. Phys. 17 (2015) 16894-16906.

[21] A.L. Sánchez, I. Iglesias, D. Moreno-Boza, A. Liñán, F.A. Williams, The slowly reacting mode of combustion of gaseous mixtures in spherical vessels. Part 2: Buoyancy-induced motion and its effect on the explosion limits, Combust. Theory Modell. 20 (2016) 1029-1045.

[22] F. Hecht, New development in FreeFem++, J. Numer. Math. 20 (2012) 251-265.

[23] B. Tyler, An experimental investigation of conductive and convective heat transfer during exothermic gas phase reactions, Combust. Flame 10 (1966) 9091.

[24] P.G. Ashmore, B.J. Tyler, T.A.B. Wesley, Experimental investigations of conductive and convective heat transfer in relation to thermal ignitions, Proc. Combust. Inst. 11 (1967) 1133-1140.

[25] J. Carpio, J.L. Prieto, An anisotropic fully adaptive algorithm for the solution of convection dominated equations with semi-lagrangian schemes, Comput. Methods Appl. Mech. Eng. 273 (2014) 77-99.

[26] S. Dennis, N. Riley, On the fully developed flow in a curved pipe at large dean number, Proc. Roy. Soc. Lond. A 434 (1991) 473-478.

[27] J.M. Potter, N. Riley, Free convection from a heated sphere at large Grashof number, J. Fluid Mech. 100 (1980) 769-783.

[28] T. Chiang, A. Ossin, C. Tien, Laminar free convection from a sphere, J. Heat Transf. 86 (1964) 537-541.

[29] F. Smith, Pulsatile flow in curved pipes, J. Fluid Mech. 71 (1975) 15-42.

[30] H. Ito, Laminar flow in curved pipes, Z. Angew. Math. Mech. 49 (1969) 653663. 